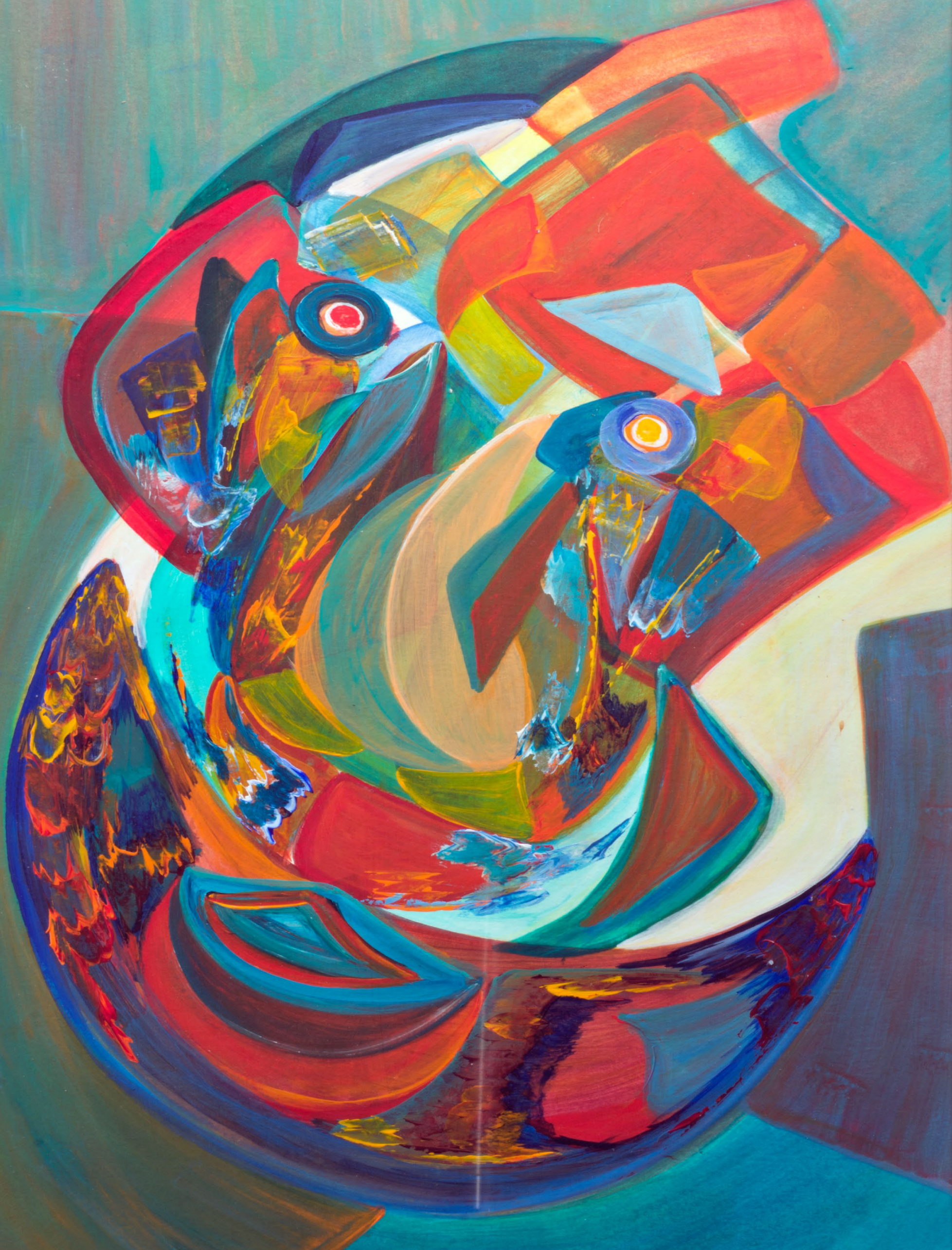




\section{Déjame nacer}

Madre... Déjame nacer,

quiero mirarme en tus ojos

para endulzar tus antojos

$y$ en tu vientre florecer.

Cúbreme con el querer, de tus maternos encantos, encomiéndame a los santos, a que protejan mi ser.

Madre, déjame nacer, arrúllame con tu canto.

Madre... quiero que me salves,

no me lances al infierno,

sabes que estoy indefenso

y frágil como las aves.

Déjame nacer no acabes, la magia de tus encantos, los destellos de mis llantos, yo quiero oír tus mensajes y sentir tus manos suaves, cual pétalos de amaranto.

Déjame nacer, te quiero,

soy el polen de tus lirios, el fruto de tus delirios,

hito de tus besos tiernos.

Quiero en el vientre materno, sentir tu voz complaciente

cuando me mimas sonriente, madre, debemos querernos

y debemos protegernos, amándonos mutuamente.

Tus encantos maternales,

te juro no me los llevo,

déjame nacery luego

no importa que me regales.

Méteme en unos huacales, déjame como un señuelo, tápame con tu pañuelo, el duro sol de la tarde, olvídate de mis males, déjame vivir... te ruego.

$80 \mathrm{cos}$

Cástor A. Olivier Olivier 


\title{
4. Adolescencia y aborto en Cuba. Percepciones de adolescentes y actores sociales
}

\author{
Luisa Álvarez Vázquez ${ }^{1}$, Aida Rodríguez Cabrera, ${ }^{2}$ \\ Nelli Salomón Avich, ${ }^{3}$, Yuri Arnold Domínguez ${ }^{4}$ \\ Recibido /8-10-2016 / Aceptado 28-03-2017
}

\begin{abstract}
RESUMEN: En la atención de salud reducir los casos de aborto es una prioridad en América Latina y el Caribe. Para disminuir esta práctica especialmente entre las adolescentes, se requiere emprender acciones conjuntas entre adolescentes y actores sociales. El objetivo de este artículo es identificar diferencias en las percepciones sobre el aborto en adolescentes y actores en el contexto geográfico de Cuba. Se analizó información cuantitativa disponible en las estadísticas continuas y resultados de las investigaciones realizadas, información de grupos focales (GF) y entrevistas en profundidad (EP), para valorar la forma de pensar de las adolescentes y otros actores sobre la decisión que conduce a abortar, así como los factores relacionados. En Cuba en los años 80 el aborto entre mujeres de 12 a 49 años fue superior a 50 por mil y aunque ha disminuido, en el 2015 aún era de 30 por mil. Las adolescentes lo practican con frecuencia y poco se conoce cómo piensa la que aborta, su pareja, y la familia. Sobre la protección relacionada con el uso de métodos anticonceptivos, aunque existe el acceso que brindan los servicios de salud, los y las adolescentes reflejan disponer de información limitada. Mientras que los actores sociales indican que los y las adolescentes no atienden las orientaciones. No se observan los resultados positivos de las acciones realizadas en sector salud y educación para evitar y reducir esta práctica en adolescentes, por un lado los y las adolescentes se consideran no informados y por otro, los actores sociales perciben que los y las adolescentes no se dejan guiar.
\end{abstract}

Palabras claves: adolescencia, actores sociales, aborto, embarazo no deseado.

ABSTRACT: Health care in reducing cases of abortion is a priority in Latin America and the Caribbean. To take action and reduce this practice, especially in adolescents, is required to achieve best results from joint actions among adolescents and social actors. The aim of this paper is to identify differences in perceptions of the abortion in adolescents and actors. Analyzed quantitative information available on the current statistics and results of investigations carried out in Cuba with information from focus groups (GF) and interviews in depth (PD), to assess the ways of thinking about the decision to abort and related factors. In Cuba in the 80s abortion among women aged 12 to 49 was superior to 50 per thousand, and although it has decreased, by 2015 was still 30 per thousand. Girls practice it frequently and little is known how he thinks that aborts, your partner and family. On the protection relating to the use of contraceptive methods, although there is the access that provide health services, the (a) teen $s$ reflect limited information. While stakeholders suggest that the (a) teen s not meet guidelines. Do not observe the actions expected results by sector health and education to prevent and reduce this practice in adolescents, I) as (a) teen s are considered uninformed and stakeholders perceived that adolescents do not let themselves to lead. (2) The lack of results of the actions may indicate that when performed is not prevention and other strategies are needed.

Keywords: Adolescence, social actors, abortion, unwanted pregnancy.

\section{Introducción}

En Cuba la práctica del aborto está legislada y el contexto social no muestra oposición, su práctica es muy especial, pues factores relacionados con las garantías para su realización reducen considerablemente los riesgos, aunque no los elimina, sin embargo, aspectos ajenos al proceder, inciden gravemente, especialmente en el caso de las adolescentes, que ocultan el embarazos, condición que puede influir para que las mujeres que lo practican tenga alto riesgo de perder la vida o que este proceder dañe su futura salud reproductiva, no solo desde el punto de vista fisiológico, sino psicológico y social (Castañeda, \& Molina, 1999; Díaz, Álvarez, \& Farnot, 2001).
Cuba presenta una evolución del proceso demográfico, caracterizada en esta etapa por niveles de fecundidad bajos (ONEI, 2009), donde la mujer y su pareja tienen la libertad de decidir libremente sobre el número y espaciamiento de los hijos.

La población cubana dispone de las condiciones para la maternidad de forma segura y sin riesgo o la regulación de la fecundidad. Cuando la mujer sale embarazada y existen razones para decidir no continuar ese embarazo, puede recurrir a la práctica del aborto. Las autoridades cubanas consideran esto como un problema prioritario de salud, en especial entre las adolescentes y brindan los servicios de salud requeridos para propiciar su mejor práctica y luchar para su prevención mediante el uso de métodos anticonceptivos.

\footnotetext{
${ }^{1}$ INEN, Demógrafa, Dra. C. Económicas, Investigadora Titular, ENSAP, Profesora Titular, La Habana, Cuba. ceicad@infomed.sld.cu

${ }^{2}$ ENSAP, Demógrafa, Dra. C. Económicas, Profesora Titular, La Habana, Cuba. aidarc@infomed.sld.cu

${ }^{3}$ Hogar materno municipal Indira Gandhi. Médico. Especialista de 1er grado en Ginecobstetricia. Master en Ciencias en Salud de la Mujer. La Habana, Cuba. salo@infomed.sld.cu

${ }^{4}$ INEN. Médico. Especialista de 2do grado en Higiene y Epidemiología. Master en Ciencias en Infectología. Profesor Asistente e Investigador Agregado. La Habana, Cuba. yuri.arnold@infomed.sld.cu ,
} 
El Sistema Nacional de Salud Pública Cubano (SNSP), tiene entre sus logros haber creado condiciones para la práctica del aborto seguro (MINSAP, 2014). Dentro del Programa Nacional Materno Infantil (PAMI) se explicita que las instituciones de salud garanticen la protección de la vida y la salud sexual y reproductiva de las mujeres que recurren al aborto. El Ministerio de Salud Pública dispone de un sistema estadístico de información de salud con datos detallados de estadísticas confiables sobre diferentes indicadores de salud, entre ellos la práctica del aborto.

En general el aborto se ha investigado a partir de lo que las mujeres que lo practican refieren respecto a ellas y sus parejas (Álvarez, 2003), pero hay elementos que indican que existen puntos de vista diferentes no solo sobre su connotación, sino sobre la participación de los miembros de la pareja y de los familiares, elementos importantes desde el enfoque de género y el entorno social para tomar medidas orientadas a la reducción del aborto. A juicio de los autores es necesario tomar en cuenta la opinión de las adolescentes, sus parejas y familiares en el diseño de acciones de salud que puede ser muy efectivo para actuar y disminuir la frecuencia del aborto, particularmente en las adolescentes. Además se intenta desarrollar nuevos enfoques estratégicos para garantizar viabilidad, aceptabilidad, eficacia y sostenibilidad, teniendo como marco normativo los compromisos establecidos por el país en la Conferencias Internacionales de Población y Desarrollo (CIPD), otras cumbres y por la Organización Mundial de la Salud (OMS), sobre la adecuada atención a la práctica del aborto, dado las características y condiciones del SNSP en Cuba.

Este trabajo se enmarca en el tema de Salud Sexual y Reproductiva (SSR) en adolescentes, tema complejo debido a su naturaleza multifactorial y a la interrelación de los ambientes familiares y sociales, así como los grupos informales de contacto, donde se refleja el punto de vista cultural y la estructura del poder predominante en la población, elementos que tienen mucha influencia en los adolescentes, que a la vez condicionan diferentes formas de interrelación según género.

El país cuenta con información para conocer la magnitud del aborto y otras características, pero muy poco se conoce cómo piensan en general los adolescentes y los integrantes del contexto social donde se desarrollan y el resto de actores que se ven involucrados en el proceso, información que puede ser de gran valor y una excelente base para identificar acciones más efectiva para disminuir el aborto.

El presente artículo tiene como objetivo identificar las per- cepciones sobre la práctica del aborto en adolescentes y actores sociales vinculados con ellas, en el país, así como considerar el enfoque de género relacionado con el aborto en la sociedad cubana.

\section{Metodología}

En el país se dispone de estadísticas continuas y de información adicional proveniente de investigaciones realizadas en distintas localidades como La Habana, capital del país y en Sancti Spiritus, provincia del centro de la isla, lugares que han aportado resultados sobre la práctica del aborto utilizando la metodología de grupos focales (GF) y entrevistas en profundidad (EP), que permiten identificar las diferencias en las formas de pensar de los adolescentes y diferentes actores sociales, sobre este problema de salud específicamente sobre la decisión para abortar y la percepción de los factores relacionados.

Las investigaciones sobre SSR han considerado las interrupciones de embarazos y el uso de métodos anticonceptivos, información que fue obtenida mediante la combinación de técnicas cualitativas y cuantitativas. Una brinda información de hombres de 20 a 29 años residentes en La Habana que participaron en GF y actores que contestaron las encuestas (Álvarez, 2004) y otras dos de adolescentes varones y mujeres de 15 a 19 añas, residentes en La Habana (Álvarez, 2015) y en la provincia de Sancti Spíritus (2013) (Álvarez \& González, 2005) que también utilizan GF, EP y encuestas. Las fuentes de información continua revisada fueron de las estadísticas continuas del MINSAP.

\section{Discusión de resultados}

El presente trabajo está dirigido a mostrar aspectos específicos relacionados con la práctica del aborto en adolescentes en el contexto social cubano. Se muestra la magnitud de esta práctica en el país y se resalta el peso y características. Se incluye una parte cualitativa relacionada con la forma de pensar de los adolescentes, así como criterios y opiniones por parte de padres, médicos, educadores y personas de la comunidad.

\subsection{Magnitud y características del aborto}

En Cuba, la tasa de aborto en los años 80 , fue superior a 50 por cada mil mujeres de 12 a 49 años. A partir de los 90 este indicador osciló entre 20 y 30 por cada mil, pero hay que resaltar que entre los años 2003 y 2005 tuvo valores por debajo de veinte (19.9 y 19.1 x 1000 respectivamente). A partir de este periodo se ven tasas oscilantes entre 22 y 26 por cada mil, pero ya en el 2013 
la cifra supera los 27 y en 2015 alcanzó el valor de 30 por cada mil, muy cercano al de la tasa del 1992, lo que constituye un retroceso en este indicador del valor registrado 20 años atrás (Gráfico № 1 ).

\section{Gráfico $\mathrm{N}^{\circ} 1$. Tasa de aborto por 1000 mujeres de 12 a 49 años,} 1980-2015.

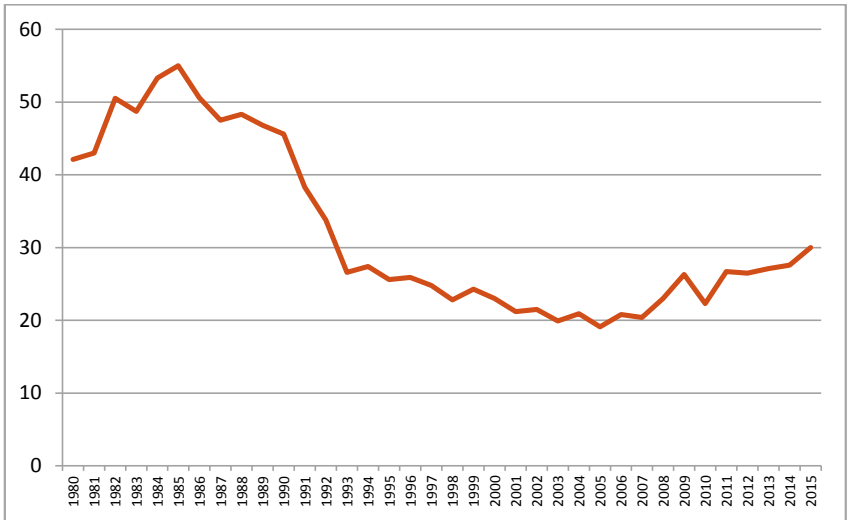

Fuente: Anuario Estadístico de Salud, Cuba, 2010-2015.

Como el nivel y la estructura de la fecundidad pueden estar muy influidos tanto por el uso de la anticoncepción como por la práctica del aborto, es importante considerar la evolución de la fecundidad en el país.

Las tasas globales de fecundidad (TGF), muestran que desde hace varios años no se alcanza el nivel de reemplazo de la población (Gráfico $\mathrm{N}^{\circ} 2$ ), esta tasa registra en 1970, 3,7 hijos por mujer y a finales de esta década comenzó un descenso marcado que hizo que en el 2015 este indicador sea de 1,72 hijos por mujer con una Tasa Bruta de Reproducción (TBR) menor de 1, propio de países que como Cuba su transición demográfica está en su última etapa (Gráfico №3).

Si se observa la evolución de la TBR (Gráfico $N^{\circ} 3$ ) y de las tasas específicas de fecundidad (de 15-19 años (TEF15-19) y de 20 a 24 años (TEF20-24) (Gráfica Nº 4), esta última sigue una evolución similar a la de TBR. Es importante mencionar que estas dos tasas específicas reflejan incrementos en el contexto del patrón de fecundidad cubano entre la población joven, incluyendo el hecho de que los niveles de fecundidad adolescente son altos (Álvarez, Rodríguez, \& Sanabria, 2000).

Los rangos de edad que reflejan mayor frecuencia de reproducción es el de 20-24 años, aunque presenta una evolución similar a la de 15 a 19 años (Gráfico № 4).

La evolución de la tasa de fecundidad de 15 a 24 años y la de aborto de todas las edades, tienen una evolución similar. Esta comparación conduce a mantener el supuesto de que el nivel bajo en la fecundidad se asocia al nivel de la práctica del aborto por las mujeres, determinante que
Gráfico $N^{\circ} 2$. Evolución de la tasa global de fecundidad. Cuba, 1970-2015.

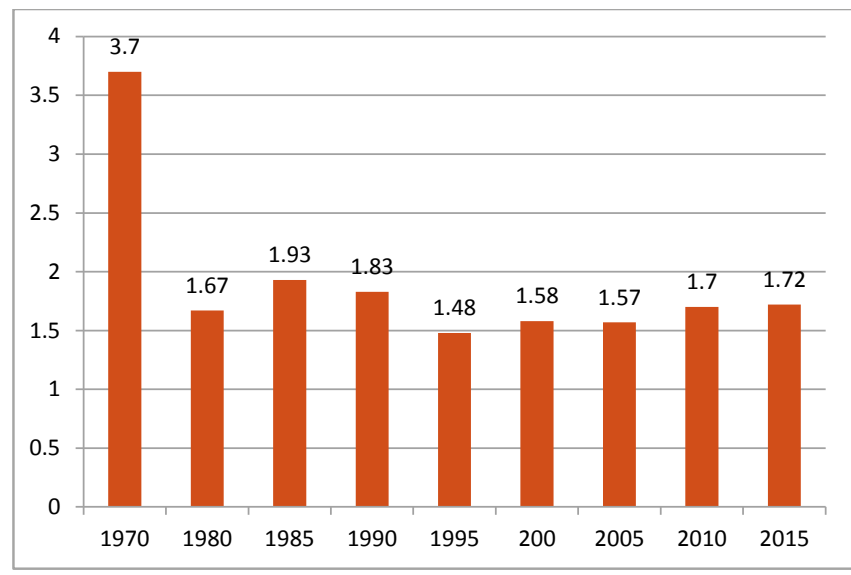

Fuente: Anuario Estadístico de Salud, Cuba, 2010-2015.

Gráfico N ${ }^{\circ}$ 3. Evolución de Tasa Bruta de Reproducción (TBR), Cuba 1980-2015

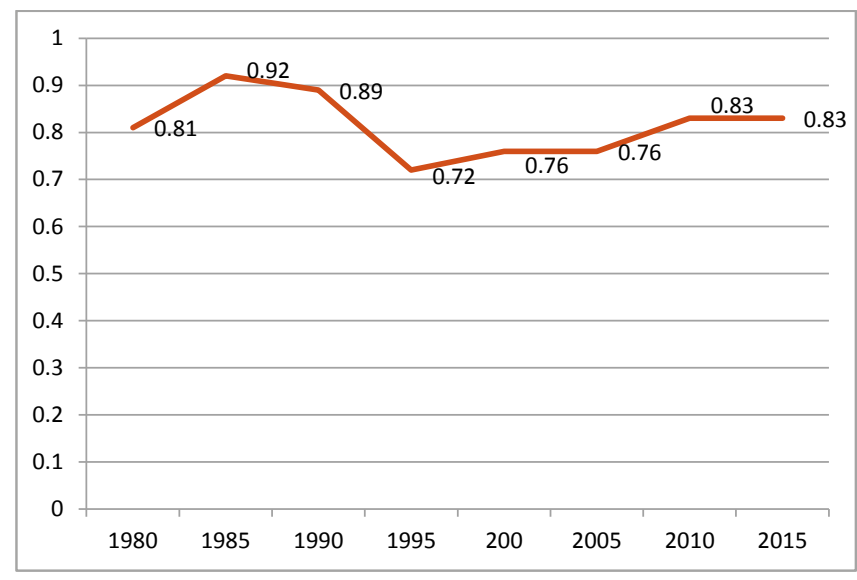

Fuente: Anuario Estadístico de Salud, Cuba, 2010-2015

Gráfico № 4. Evolución de las tasas especifica de 15-19 y 20-24 años, Cuba. 1980-2015

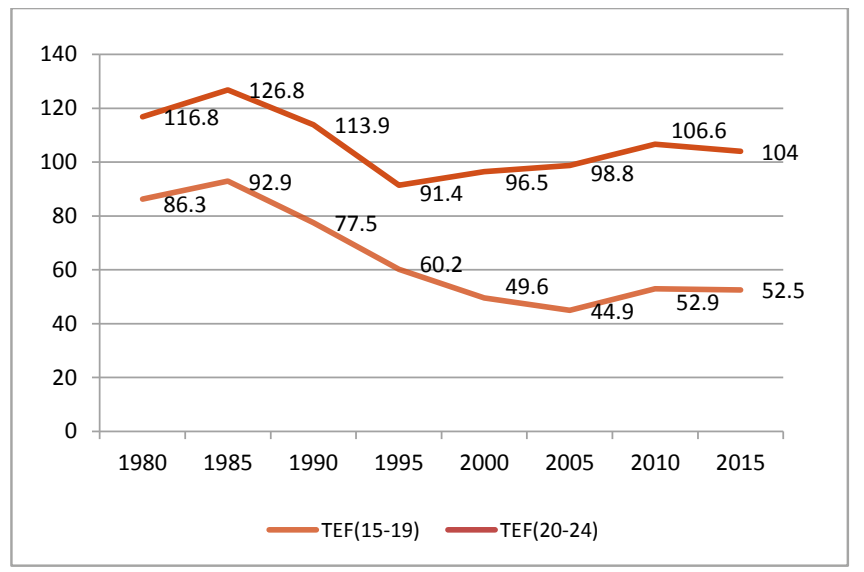

Fuente: Anuario Estadístico de Salud, Cuba, 2010-2015.

más aporta a la reducción de la fecundidad (Gráfico $n^{\circ} 5$ ). La evolución de la tasa de fecundidad de 15 a 24 años 
y la de aborto de todas las edades, tienen una evolución similar. Esta comparación conduce a mantener el supuesto de que el nivel bajo en la fecundidad se asocia al nivel de la práctica del aborto por las mujeres, determinante que más aporta a la reducción de la fecundidad (Gráfico $n^{\circ} 5$ ).

\section{Gráfico № 5 . Evolución de Tasa Especifica de Fecundidad de 20-24 años y la Tasa de Aborto, Cuba 1980-2015}

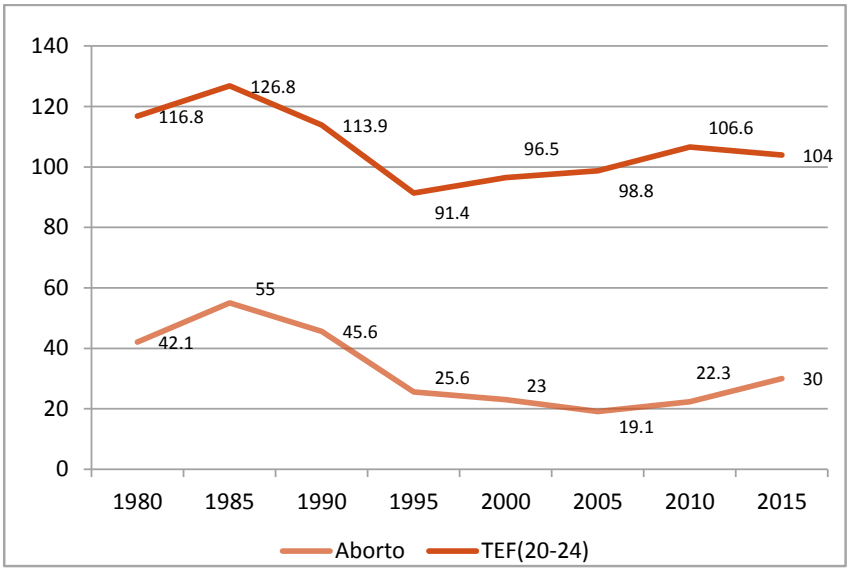

Fuente: Anuario Estadístico de Salud, Cuba, 2010-2015.

\subsection{La percepción de los y las adolescentes sobre el aborto}

Para conocer la forma de pensar de los adolescentes se debe comenzar por conocer lo que ellos refieren sobre la protección en las relaciones sexuales con penetración.

La investigación realizada en el municipio 10 de octubre (La Habana), indica que $68.9 \%$ de las adolescentes refieren haberse protegido en sus relaciones sexuales. En relación a la decisión de usar protección en el $57.7 \%$ de los casos el hombre decidió la protección, y $68.7 \%$ de los métodos anticonceptivos eran de uso masculino. EI $55.0 \%$ de los adolescentes varones, refirió haberse protegido y $13.3 \%$ no dio respuesta. Entre las respuestas se identifica el hecho de que ambos lo acordaron (33.3\%). Sobre esta misma pregunta el $90 \%$ de las mujeres que se protegieron indicaron que la decisión fue del hombre y de ellos $63.6 \%$ con métodos masculinos.

Las razones fundamentales para no usar los anticonceptivos según las mujeres fueron: creer que no iban a tener problemas $(40.5 \%)$ y no tener condiciones para usar la protección (31.0\%). Estas dos razones reflejan la falta de responsabilidad que tienen los jóvenes en relación a la práctica de relaciones sexuales y el inminente riesgo de un embarazo.

Por su parte los hombres identificaron razones como: que a ellos no les gusta eso, que su pareja no quería usar nada y que creían que no iba a pasar nada, esta última razón es similar a la dada por las mujeres.

Cerca del $50 \%$ de los hombres consultados en La Habana expresaron que no se cuidaron, dando como razón fundamental de ello "no lo planearon" y "se conocían". Entre los que se protegieron tres quintas partes indicaron que fue para "evitar embarazos", una cuarta parte para "cuidarse del ITS/SIDA", un $14 \%$ lo hizo por ambos motivos. Los hombres manifestaron que no hablaron con la pareja sobre protegerse, y el $26.3 \%$ de ellos adujeron como razón fundamental que "no acostumbran a hablar del tema".

En relación con el proceso de toma de decisión sobre el uso de protección al momento de una relación sexual en los GF, se nota un ligero predominio de la mujer, pues en la investigación de Santi Spiritus fue mayor la proporción donde ella la propuso (31.3 \% contra 26.6 \%). En los casos en que el encuestado señala que él propuso la protección, la pareja por lo general estuvo "de acuerdo". Aparentemente cuando un miembro de la pareja propone la protección el otro está de acuerdo (92.7\%).

Respecto a cómo se expresan los/las adolescentes sobre la protección en un GF de mujeres las ideas contra el uso de métodos de planificación familiar se rescatan las siguientes ideas:

"Porque los varones dicen que ellos no sienten nada, como ellos no sienten nada? La mujer que no, yo no sé, y actualmente como las mujeres, y las muchachitas se dejan guiar por el pensamiento del hombre, no, él es el hombre y es el que decide, entonces si él no quiere, ella que va a hacer" (Muchacha de 17 años, Sancti Spiritus).

“...que yo no tengo ninguna enfermedad, si- eso es lo que ellos no saben-pero, eso no es por desconfianza, sino por protección". (EP adolescente de 16 años $10^{\circ}$ ).

“...también hay muchachas que no lo utilizan porque dicen, no porque es más rico a capela, si porque yo digo lo que yo oigo, el lenguaje de la calle, entonces dicen que yo no compro eso". (Muchacha habanera de 17 años 11 no. Grado)

"Yo pienso que eso es parte de la incultura tienen actitudes machistas no, no me pongo el condón porque eso es cosa de homosexuales, porque yo soy hombre y yo no uso eso" (Adolescente de 16 años con 9no grado terminado). 
Todas estas expresiones se pueden resumir en la idea de que las adolescentes consideran que es el hombre el responsable y dominantes en la relación sexual.

Los criterios de adolescentes varones o de hombres pareja de las adolescentes indican otro pensamiento sobre el uso 0 no de la protección. En las entrevistas a profundidad dirigidas a los varones en La Habana se rescatan algunas ideas:

"Ella también aceptó a mí no tiene que preocuparme, si yo no tengo un trato con esa muchacha, que salga embarazada o no, a mí no me preocupa, si ella no se preocupa es su problema, eso es otra cosa si yo no la conozco". (Joven de 19 años).

"No tanto para los varones como para las mujeres, que pueden tener un embarazo precoz, no deseado, y también hay veces que nosotros mismos nos limpiamos las manos y ya. Sí que le damos de lao al problema, y que ella sola lo resuelva" (Joven de 15 años y de 9no grado)

Todo esto nos lleva a plantear que el varón considera que el aborto es problema de la mujer; en este caso ellos parten que fue ella la que no se protegió y en los casos en que se refiere a una pareja no estable, si se embaraza o no, consideran que la responsabilidad es de ella y de su familia.

Otro aspecto de importancia es lo que conocen sobre su protección sexual, los adolescentes refieren que no reciben información al respecto.

"Bueno, el que enseña eso son los padres y mi papá siempre estuvo fuera y no pudo, nunca he tenido conversación acerca de eso normalmente," Sobre los amigos dice "No, no es que me digan ellos a mí, sino que yo les cuente siempre lo he hecho sólo, nunca he hablado con nadie, me informo eminentemente solo, solo. Yo siempre estoy, me gusta estar informado, me gusta saber de todo" (Adolescente varón de 15 años).

"Como que porque tengo 16 años, pero como que nunca me han hablado de esas cosas, mi mamá ha hablado, pero no así, he recibido información de amigas, de mi familia y en la escuela nos hablan no sólo con respecto a esto, sino también por las drogas, que no debemos tener" (Adolescente mujer 16 años).

De los diversos factores que pueden ser valorados para lograr disminuir la práctica del aborto está evitar el embarazo, razón por lo cual en el análisis se ha hecho énfasis en factor.
Al considerar el tema del aborto entre los adolescentes, se debe destacar que la base fundamental son los embarazos no deseados, y el enfoque de género está relacionado con ello, pues en la adolescencia predominan elementos multifactoriales entre ellos el embarazo que en su mayoría son no deseados por la adolescente o por su familia.

Por otro lado en el adolescente varón predomina la concepción social de que no es problema de ellos. De aquí que, para la adolescente embarazada, abortar es una práctica altamente considerada y el que el sistema de salud garantice el aborto en excelentes condiciones hace que sean factores determinantes de la elevada proporción que considere el aborto como la mejor solución ante un embarazo no deseado. Otros factores como problemas de salud y riesgos de tener hijos posteriores tienen menos peso entre las adolescentes y la familia que apoya esta acción.

\subsection{Opiniones y criterios de los actores sociales sobre el aborto en adolescentes.}

Cuba está entre los países, donde el Estado garantiza la realización del aborto a las personas con las condiciones adecuadas para practicárselo, cuando esto sea solicitado, pues se parte de lo que señala la Organización de Naciones Unidas (ONU), que garantiza el acceso a este servicio con calidad y gratuidad, lo que está en conformidad con los estándares de derechos humanos. La despenalización del aborto como derecho exige la garantía de una práctica segura y con el mínimo de riesgo, por lo que el Estado cubano garantiza las condiciones en los servicios, al disponer de profesionales entrenados para su realización y exigir que se cumplan lo requerido para que haya un riesgo mínimo para la salud de la mujer.

Entre las actividades que se deben garantizar, para lograr que esta práctica sea sólo una opción alternativa para la mujer, es que todos los involucrados directa o indirectamente conozcan los aspectos más relevantes del mismo. Aunque el MINSAP realiza acciones, no existen estudios que muestren los logros, en este sentido, el presente estudio trata de llenar el vacío existente.

En la investigación sobre adolescentes, se entrevistaron distintos actores sociales relacionados con los adolescentes, la mayoría fueron madres (55.8\%), y la menor cantidad padres (6.9\%), además de incluir a estos, también consideraron a miembros de la comunidad, del sector salud y de educación.

“La familia de la niña, no sé, la sobreprotegemos

Revista Población y Desarrollo: argonautas y caminantes 
más, la queremos para nosotros, pero la familia del varón, por lo general no asume" (Madre entrevistada en Sancti Spiritus).

El resultado a resaltar sobre los criterios emitidos por los actores sociales fue que los adolescentes no los escuchan.

"Muy poco, muy poco se guían, prefieren comentárselo a una amistad, a un tío, a una abuela, no sé. Mi hijo si habla conmigo, otros no. Mi hijo tiene una amistad, que va y me cuenta las cosas a mí en vez de su mamá. Bueno, las hembras tienen una característica diferente, prefieren muchas contar con la amiguita, no se dirigen a los mayores. Prefieren muchas contar con la amiguita y no con la mamá" (Madre de un varón habanero de 16 años).

Estudios anteriores indican que los actores sociales que conviven con los adolescentes tienen limitaciones para hablar con los adolescentes, pues aun cuando todos valoran la importancia del tema, no se hacen responsables uno a otros de la tarea.

Entre los trabajadores de salud, el problema del aborto está priorizado y existe un programa llamado Programa de Atención Materno Infantil (PAMI), que plantea medidas para evitar el aborto.

"Mira, antes las pacientes llegaban, el médico le hacía un tacto, un reconocimiento clínico y ahí detectaban el embarazo, ahora las mujeres vienen directamente solicitando un ultrasonido para que les detecten el embarazo porque no quieren hacerse el tacto. Ya lo piden. Entonces la decisión la toman después que se hacen el ultrasonido. Al médico no le queda ni siquiera la oportunidad de hacer la pregunta, pero por otra parte llegan directamente diciendo: "Doctora, estoy embarazada y me lo voy a interrumpir", aunque es verdad que el médico no está exento de una formación en la cual las mujeres tienen una cultura abortiva" (Dirigente del PAMI*).

"Yo pienso que nuestra población es una población bien informada pero carente del conocimiento necesario para hacer un uso adecuado de esa información. En nuestra experiencia médica está reflejando eso. Cuando se empieza a concientizar en la población la problemática del SIDA y las ITS se aumenta el uso real del condón, ahora nunca se ha concientizado en la necesidad de usarlo para proteger contra un embarazo no deseado, porque el uso del condón para la protección contra un embarazo requiere de técnicas de su uso y esta técnica se obtiene de conocimiento y ese conocimiento la gente no lo tiene" (Dirigente municipal del PAMI).

El sector de educación también les preocupa el problema, una profesora expresó lo siguiente:

“... A la muchachita si la cuidamos, si le decimos que debe protegerse de un embarazo, entonces esa no es preocupación por parte del hombre, el no inmiscuirse en la decisión tiene sus raíces como hemos visto mucho más allá, en la propia educación, en los patrones sociales" (Profesora de Secundaria Básica, La Habana).

En general la población conoce los aspectos del aborto e identifica los problemas fundamentales como se ve en una discusión grupal de personas de la comunidad:

"En las adolescentes hay también un fenómeno, ocultan más tiempo el embarazo y por tanto, en mayor medida recurren a la interrupción y no a la regulación menstrual, y ahí caes en el tema de la relación entre madre y padre con los hijos, la familia, porque si ustedes fueran a hacer una investigación sobre la detección tardía del embarazo se darían cuenta de cuantas adolescentes tenemos que conocemos de su embarazo a las 18 semanas, y entonces ya el tema de la interrupción es otro, porque hay que tener claro que en Cuba el tema de la interrupción del embarazo es un derecho libre de la mujer hasta las 10 semanas, porque a partir de ahí entonces ya es decisión médica" (Hombre, trabajador).

En los últimos años las tasas de aborto en Cuba han disminuido, pero esta sigue siendo alto y tiene gran peso en la disminución de la fecundidad. El descenso brusco de esta tasa en los años noventa, indica la necesidad de indagar sobre qué acciones pueden estar asociadas. Se encontró que desde el 1989 en Cuba comenzó a utilizarse una forma menos invasiva de interrupción de un posible embarazo, pero sin confirmación, este método es llamado "regulación menstrual", los que en su mayoría son interrupción de embarazos (Gran, 2001).

Este supuesto permite considerar otra información presente en este trabajo, como es la evolución del nivel de la fecundidad y el comportamiento de las tasas específicas de fecundidad en el grupo de adolescentes y en el grupo de máxima fecundidad por edad. La evolución de los niveles de la fecundidad están altamente relacionadas con la tasa de fecundidad (TEF20-24.). Sin embargo, el elevado nivel de la tasa de 15 a 19 años (TEF15-19), no tiene la 
evolución esperada con el bajo nivel de fecundidad (MINSAP, 2016).

El comportamiento de esta tasa puede estar relacionada con el proceder iniciado estos años de la regulación menstrual y que por tanto esa evolución indica la subestimación de las tasas de aborto en la referida época (Domínguez, Puentes, \& Bustamante, 2012; Álvarez, \& Salomón, 2012).

Dado que en el país el uso de métodos anticonceptivos (ESTADISTICSALUD) y la práctica del aborto tienen gran peso y son determinantes del bajo nivel de fecundidad, estos resultados hacen pensar que esta disminución del aborto, se debe al aumento de las regulaciones menstruales y por tanto existe una subestimación de las cifras de aborto, sobre todo en las adolescentes del país.

En estudios anteriores los resultados encontrados permiten resumir que un número elevado, tanto de adolescentes varones, como mujeres, refieran causas inconsistentes 0 sea expresiones no concretas sobre la razón para protegerse o no hacerlo, esto indica que, aunque se protejan, no tienen idea clara de porque lo hacen, lo que constituye un riesgo latente para abandonar la protección. En el grupo de hombres que se entrevistó en La Habana, la protección en la relación sexual supone el predominio del machismo respecto a la práctica sexual, de forma similar que en trabajos anteriores.

En estudios anteriores que consideran la relación entre la información dada sobre si se protegieron o no y la relativa a la conversación con su pareja sobre la protección, está claro que la mayoría de los que no conversaron consideraron que no hubo protección, lo cual puede o no ser cierto. Lo importante de esta información es que, en muchos casos, la decisión de la protección se deja como responsabilidad del otro miembro de la pareja y no se llega a tener plena conciencia que es una responsabilidad de ambos.

En la investigación de adolescentes habaneros, y de otros lugares del país, estos no identifican que reciben la información necesaria, posiblemente se le brinda parte de esta información, pero la captación se percibe insuficiente y las actitudes y conductas muestran una falta de dominio para evitar la excesiva práctica del aborto (Domínguez, Puentes, \& Bustamante, 2012; Álvarez, \& Salomón, 2012).

Respecto a la orientación y participación de los actores sociales sobre el embarazo en la adolescencia, los estudios muestran que muchos actores sociales sienten que estos problemas no se asocian a ellos y la participación para su posible solución difiere entre ellos.

En este estudio, se constata las diferencias en el papel de los actores sociales para la toma de decisión respecto a la práctica del aborto por la adolescente, donde la madre participa activamente, pero, otros actores sociales no intervienen. En un artículo publicado en el 2010, sobre los resultados de una investigación en el servicio de aborto del hospital "Dr. Julio Rafael Alfonso Medina" de Matanzas (Doblado, De la Rosa \& Junco, 2010), se refleja nítidamente esto cuando refieren: "cuando evaluamos la variable toma de decisión sobre el aborto vemos que el $69,5 \%$ de los casos fue la madre la que tomó la decisión de interrumpir el embarazo, y en el $62 \%$ fue la propia paciente la que optó por el aborto, sin embargo solo en el $7,8 \%$ de los casos la pareja tuvo que ver en la decisión".

\section{Conclusiones}

- El proceso de toma de decisiones para el aborto entre las adolescentes se apoya más en las madres que en otros actores sociales. Se identifican diferentes formas de percibir la responsabilidad para recurrir al aborto, según sean adolescentes varones o mujeres la que lo expresan.

La práctica del aborto está basada en normas tradicionales aun presentes en el medio familiar y en el ambiente social, por lo que estas juegan un papel fundamental en la forma de actuar de la adolescente. Un detalle importante es que los adolescentes no se consideraran informados y los actores sociales perciben que los adolescentes no se dejan orientar.

La falta de resultados de las acciones para disminuir el aborto puede indicar que cuando estas se realizan no se logra la prevención y se necesitan otras estrategias.

\section{Bibliografía}

Aborto en Cuba (2014). Wikipedia. doe: https://pt.wikipedia.org/wiki/ Aborto_em_Cuba

Mayo, D (2002). Algunos aspectos histórico-sociales del aborto. Revista Cubana de Obstetricia y Ginecología. 28( 2): 45-6.

- Castañeda, I., Molina, E. (1999) Factores biosociales que influyen en la aparición del aborto provocado. Facultad de Salud Pública. Rev Cubana Obstetricia Ginecología, 1999;25(1):55-60

Revista Población y Desarrollo: argonautas y caminantes 
Díaz, E., Álvarez, L., Farnot, U. (2001) Factores de riesgo demográficos y sociales del aborto. Revista Cubana Salud; 27(1): 26-35.

ONEI. (2009). La fecundidad en Cuba: Momentos significativos, ONEl, 1963-2008. Recuperado de: http://www.onei. $\mathrm{cu} /$ publicaciones/coleccionestadisticas/Fecundidad.pdf

MINSAP. (2014) Programa Nacional Materno Infantil de Cuba, 2013. La Habana. Recuperado de: https://www.ecured.cu/Programa_Materno-infantil_en_Cuba

Álvarez, L. (2003). El aborto: ¿Un problema de salud en Cuba? En: Planell E. Silencios, presencias y debates sobre el aborto en Puerto Rico y el Caribe hispano. Puerto Rico: Fundación Atlantea: pp:34-78

MINSAP (2016). Anuario Estadístico de Salud. Cuba, 2015. 1ra edición, pp: 166, 197.

Álvarez, L. (2004) Percepciones y comportamientos de la sexualidad y la reproducción según los hombres. La Habana: Publicaciones Azucareras. La Habana, pp: 13-89.

A Álvarez, L. (2015) Informe final de la investigación:Atención Integral de adolescentes y prevención de la Salud Sexual y Reproductiva con enfoque de género. La Habana, La Habana, pp 34.

Álvarez, V., González, V. (2005) "Percepciones y comportamientos en adolescentes: Una perspectiva de la salud reproductiva por género" Informe Final de Investigación", pp:23-45

Álvarez, L., Rodríguez, A., \& Sanabria G. (2000). Coordinadoras: Salud sexual y reproductiva en adolescentes cubanos. La Habana: Casa Editora Abril; p: 56-9

Gran, MA (2001): Interrupción voluntaria de embarazo y anticoncepción: dos métodos de regulación de la fecundidad: Cuba 1995-2000, pp: 34-86.

Domínguez, BE., Puentes, E., Bustamante, D. (2012). Comportamiento de la regulación menstrual en la adolescencia. Rev Cubana Med Gen Integral 28 (4)

Álvarez, L. Salomón, N. (2012) El aborto en adolescentes en un contexto legal. Rev Cubana Salud Pública, 38 (1): 4553.

Doblado, D., De la Rosa, B., Junco, M. (2010). Aborto en la adolescencia un problema de salud. Revista Cubana de Obstetricia y Ginecología. 36(3) 409-421 